\title{
The views and attitudes of general practitioners and smokers toward provision of smoking cessation advice: a qualitative study
}

\author{
Jennifer Cleland*, Mike Thomas, Hilary Pinnock
}

\author{
Department of General Practice and Primary Care, Foresterhill Health Centre, Westburn Road, \\ University of Aberdeen AB25 2AY, UK
}

Received 13 November 2003; accepted 6 May 2004

\section{KEYWORDS}

Smoking cessation;

Primary care;

GP views;

Patient views;

GP-patient concordance

\section{Introduction}

Smoking is the single greatest cause of preventable illness and premature death in the UK [1]. Treating illness and disease caused by smoking is estimated to cost the NHS up to $\mathrm{f} 1.7$ billion every year [2]. The success, or otherwise of smoking cessation programmes will significantly influence mortality in the

*Corresponding author. Tel.: +44 1224553969.

E-mail address: jen.cleland@abdn.ac.uk (J. Cleland).
Summary Objectives: To identify common themes between general practitioners (GP's) and patients on smoking cessation in primary care in order to inform the development of acceptable guidelines, thus maximising the chance that recommendations will be received positively and implemented.

Design: Qualitative study using focus groups and individual interviews with GPs and patients.

Setting: North East Scotland.

Subjects: 10 general practitioners and 20 patients ( 10 smokers and 10 patients who described themselves as ex-smokers).

Results: Both general practitioners and patients agreed that the GP has a key role in providing a range of advice and support for smoking cessation. Both parties expressed views at variance with current guidelines but agreed that, for support and advice to be successful, it needs to comply with four of the five main themes identified; that it should be practical, pertinent to the consultation, personalised to the smoker's clinical need, and should emphasise the positive health gains from quitting.

Conclusion: The considerable concordance between the perceptions of GPs and their patients about smoking cessation care suggests potential for a more positive partnership in working towards reduction of smoking in the UK.

(c) 2004 General Practice Airways Group. Published by Elsevier Ltd. All rights reserved. first half of the 21 st century, emphasising the importance of offering effective support and advice [3].

The White Paper "Smoking Kills" promoted the evidence-based recommendation that general practitioners (GPs) should adopt a population-based approach to providing brief, opportunistic smoking cessation advice [4]. This was further endorsed by the 1998 British Thoracic Society (BTS) smoking cessation guidelines [5] and their subsequent update [6]. That less than half of smokers remember being given advice in the previous five years suggests that these guidelines have had little impact $[7,8]$. 
Despite commitment to health promotion [9], general practitioners cite many barriers to incorporating smoking cessation activity into routine general practice, such as reluctance to jeopardise the doctor-patient relationship [10]. Crucially, guidelines may recommend an approach that does not concur with GP attitudes towards healthcare. Evidence from selected research populations may not be seen as applicable to unselected patients in general practice in terms of levels of co-morbidity [11]. The short-term follow-up in many studies may compromise the validity of recommendations and further reduce GPs willingness to adhere to guidelines [12].

Similarly, we know that tobacco users' attitudes and beliefs influence their willingness to adhere to advice from their doctor [13]. Tobacco users may resent unsolicited health promotion particularly if this is presented in an unacceptable manner, such as not acknowledging the patient's perspective [14].

Recognition that GPs and smokers are more likely to adhere to smoking cessation guidelines if they concur with the advice given, [15] should encourage the integration of attitudes and perceptions into their recommendations [16]. This study aimed to identify areas of common ground between GP and patient attitudes to inform the development of acceptable guidelines, thus maximising the chance that recommendations will be received positively and implemented.

\section{Methods}

The study, which had local research ethics committee approval, was undertaken during 2002.

We used two complementary qualitative methods - focus groups and in-depth interviews [17]. Focus groups give participants the opportunity to set the agenda and are recognised as being a particularly valuable method for investigating what people think and why, but can sometimes be dominated by certain participants [18] Thus, individual in-depth interviews were also carried out. We purposively sampled (practice location, age and gender) ten GPs and twenty patients from four practices reflecting the urban (Aberdeen)/rural divide in North East Scotland. None of the doctors interviewed expressed a special interest in tobacco control.

Participants were given the choice between taking part in a focus group or interview. We carried out one focus group (6 people) per category of participants (GPs, smokers and ex-smokers) and in-depth interviews with four additional people from each category. Patients were recruited from the prac- tices of participating general practitioners and reflected the socio-economic profile of the area.

Questions to use as a guide for discussion were developed by the authors, in conjunction with staff from the Smoking Advisory Service, Grampian NHS. Sessions were audio-taped, with permission.

The focus groups and interviews were carried out by a non-medical/social science research assistant from the Department of General Practice and Primary Care.

\section{Analysis}

Tapes were transcribed. Data analysis followed accepted qualitative analytic methods of inductively developing higher order themes (by JC and the research assistant [19]) from open coding of the transcripts. Codes were developed independently using constant comparative methods, by one author (JC), the research assistant and an independent researcher, who then compared analysis and identified five main themes using the software package Nvivo to manage the data. These were: the use of guidelines, the need for practical support, the advice being pertinent to the subject of the consultation, the timing of the follow-up advice being personalised to the individual patient, and the need for the GP to give positive information as part of the smoking cessation service.

\section{Results}

\section{Use of guidelines}

None of the GPs were familiar with national guidelines on the provision of smoking cessation advice and support.

"I think the difficulty is that we are swamped by guidetines... to implement fifty that are all coming in .. that'swhen it gets a bit more difficult" The GP focus group members pointed out that there are no SIGN guidelines (Scottish Intercollegiate Guidelines Network: an evidence-based group http://www.sign.ac.uk) specific to smoking cessation advice and support in primary care. Notwithstanding this, the GPs were confident that they had an important role in helping patients to stop smoking:

"I think the GP has a major role in it. I think the one-to-one contact and particularly when it is associated with health risk factors then I think you can have a lot of input" 
Time constraints meant that GPs appreciated the local provision of a specialist smoking cessation service to which patients could be referred.

"I think the Smoking Advice Service is very good to give the ongoing counselling that we do not have time for ..."

However, they recognised the need to offer a flexible service to meet the needs and preferences of all their patients.

"you have to be able to tailor different methods for different people"

Smokers agreed and generally expressed positive attitudes towards such agencies, though did not necessarily think they were appropriate for themselves.

"I think a lot of these things. I mean they are good for someone who has maybe been smoking for years and years but if you are young enough I think you can really stop if you want." [Smoker]

Paradoxically the Smoking Advisory Service, and local guidelines were perceived by GPs as barriers to providing timely and individualised support.

" ... the LHCC [Local Health Care Co-operative] guideline is for us not to prescribe bupropion or NRT [nicotine replacement therapy] until the person has been through the Smoking Advice Clinic which feels like a bit of a handcuff at times, not being able to start something when people are motivated ..."

\section{Practical support}

Smokers and their GP acknowledged the importance of providing practical help.

"It would have been better if somebody had sat down and explained how to stop smoking ... most people know what smoking can do to you anyway ..." [Ex-smoker]

\section{Pertinent to the subject of the consultation} Doctors, smokers and ex-smokers were united in their opinion that there was a time and a place for smoking cessation advice.

"... there are some times when it is far more appropriate to ask and some times it's totally inappropriate so you know certainly if someone comes in with any respiratory tract infection ... you can target the smoking in" [GP]

" ... if you were in for something else, you don't want the GP to be telling you to stop smoking, you just want your prescription for whatever." [Smoker]

In consultations not pertaining to smoking-related disease, GPs were aware of the need "to be sensitive to the consultation' ' and the patients agreed that opportunistic advice was unlikely to be helpful.

"... most people who smoke won't want advice anyway but it is up to doctors to try..." [Ex-smoker]

Smokers were resigned to being asked about their smoking as part of health check ups, though it was not necessarily effective.

"I think that I did get asked once on a check-up if I actually smoked. Apart from that I can't say ... I smoked at the time so it was in one ear and out the other."' (Smoker)

\section{Personalised to the individual patient}

Making a link between the patient's own symptoms and smoking was seen by both GPs and smokers to be important, even encouraging.

"... I fainted one morning ... and had to go to the doctor ... and he knew I took an occasional cigarette and he said 'you know, xxxx, you would be better if you stopped taking any at all', and that was really when I stopped" [Ex smoker]

GPs thought an acceptable approach was to enquire if the patient had thought about stopping, then tailoring their response to the patient's reply. For example:

"... you are trying to assess where they are on the change cycle. If they are not, if they feel they are not ready to do anything, then that is the end of it"'

GPs recognised that if a patient was not ready to quit at that time, it was important to invite them to seek advice later:

"If they are not interested then I'll just acknowledge that and say 'Maybe come back to it someday'.", [GP]

This was reflected in the views of the patients:

"... I think you can only do it when you're ready to do it yourself. . . ... If you're not ready to stop, you don't want to be harassed"' [Smoker]

\section{Positive information}

While patients recognised the role of the GP in providing general advice, advice on the adverse effects 
of smoking was seen as more useful if linked to positive benefits to the individual:

"...I think being told you can benefit from not smoking would make you come out and smile and think 'well, maybe I will give it a go, try and stop' or whatever, so I think that is maybe a better way to go about it"' [Smoker]

\section{Discussion}

The considerable concordance between the perceptions of GPs and tobacco users about acceptable smoking cessation advice suggests potential for a more positive partnership in working towards reduction of smoking. Both parties expressed views at variance with current guidelines but agreed that successful support and advice should be pertinent to the consultation, personalised to smoker's clinical need, should emphasise the positive health gains from quitting, and should be practical and timely, including, where desired, ongoing support and pharmacological treatment rather than the only option being referral to a specialist service.

\section{Limitations and strengths of our study}

This small study sampled GPs and patients from one area within the UK and some comments, particularly those reflecting local services, may be unique to a specific area of the UK, though local guidance is normally based on national policy and guidelines. By interviewing patients and GPs from the same practices we were able to compare two perceptions of the same smoking cessation care.

\section{Interpretation of findings in relation to previously published work}

The behaviour of the GPs in practice seems to be based on a desire to work in partnership with the patient [20-22] thus avoiding risking damage to the doctor-patient relationship, as wellas factors identified by previous studies such as time constraints [23], rather than strictly adhering to guidelines even those which recognise the need to integrate user and professional perspectives when making guideline recommendations (e.g., NICE http://www.nice.org). Thus the pragmatic stance that smoking cessation should normally only be discussed in clinically relevant situations suited both parties. This discrepancy between the efficacy of an intervention in clinical trials and the practicability in pragmatic studies has recently been described in the context of screening and brief advice for excessive alcohol use. [24]
Both parties agreed that advice should be personalised to the smoker's clinical condition and tailored to individual needs [13]. Smokers wanted more information about the health gains, a positive motivation to quit, from smoking cessation. Practical support should be timely and readily available; that is, when the smoker is ready to quit. Linking advice to the patient's personal timetable suggests that GPs may benefit from having more training in objective ways of identifying how ready smokers are to stop.

Local health service dictates restricting the role of the GP and the use of pharmacology were perceived as barriers to patient-centred care and may be a counter-productive way of rationing, particularly as NICE [25] describes bupropion and nicotine replacement therapy as among the most cost-effective of all healthcare interventions. The views of our patients and GPs suggest that a sliding scale of service provision, where GPs are able to provide a range of smoking cessation services rather than just being able to offer simple advice or specialist referral, would be preferable. [26]

It is disappointing that GPs in this study were unaware of guidelines for the provision of smoking cessation in primary care. It is recognised that GPs may feel inundated by guidelines and seem to have made a pragmatic decision to focus on those from one recognised national source, despite the restrictions this imposes.

Whilst randomised controlled trails and systematic reviews are the best methodology we have for establishing basic clinical and pharmacological effectiveness, they provide limited information about the general applicability of results to the general, primary care population. The updated BTS guidelines [6] acknowledge the contributions of studies that address the clinical and economic realities of the "real world" and reflect a welcome progression in guideline development which may encourage positive attitudes to new guidelines and aid their successful implementation in practice.

Our results, and those of other authors, may underpin the next stage in further determining the information needed to inform the development of acceptable guidelines - a questionnaire survey.

Funding: Chief Scientist Office: Grant no CZG/4/ $2 / 52$

\section{Statement(s) of conflict of interest}

None known 


\section{Acknowledgements}

Professor David Price for his valuable comments on this, and earlier drafts of the paper. Dr Scott Lennox and Professor Thys van der Molen for their helpful input into the initial discussions. Mrs Jan Caldow, for her invaluable work. Finally, the GPs and patients without whom this study could not have taken place.

$\mathrm{JC}$ and SL led the development of the protocol and securing of funding. JC was responsible for the management of the study and the researcher, and for the production of the CSO project report and the final draft of the paper. JC, HP and MT were involved in the development of the protocol, interpretation of results and writing of previous drafts of the paper. All authors reviewed the final manuscript. JC is the study guarantor.

\section{Presentations}

This work has been presented at the BTS Winter Meeting, London, 3-5 December 2002, and the American Thoracic Society, Seattle, 17-21 May 2003.

\section{References}

[1] Doll R, Peto R, Wheatley K, Gray R, Sutherland I. Mortality in relation to smoking: 40 years' observations on male British doctors. Br Med J 1994;309:901-11.

[2] Buck R, Peto R, Wheatley K, Grey R, Sutherland I, University of York Centre for Health Economics. Cost effectiveness of smoking cessation inerventions. 1997.

[3] Peto R, Darby S, Deo H, Silcoks P, Whitley E, Doll R. Smoking, smoking cessation and lung cancer in the UK since 1950: combination of national statistics with two case-control studies. Br Med J 2000;321:323-9.

[4] Kottke TE, Battista RN, DeDriese GH, Brekke ML. Attributes of successful smoking cessation interventions in medical practice. A meta-analysis of 39 controlled trials. JAMA 1988;259:2883-9.

[5] British Thoracic Society. Smoking cessation guidelines. Tho$\operatorname{rax} 1998 ; 53(\mathrm{~S} 5): \mathrm{S} 1-\mathrm{S} 19$

[6] West R, et al. Smoking cessation guidelines for health professionals: an update. Thorax 2000;55:987-99.

[7] Baker R. Is it time to review the idea of compliance with guidelines? Brit J General Pract 2001;51:7.

[8] Freeth S. Smoking-related behaviour and attitudes, 1997: a report on research using the ONS Omnibus Survey produced on behalf of the Department of Health. 1998.
[9] McAvoy BR, Kaner EF, Lock CA, Heather N, Gilvarry E. Our Healthier Nation: are general practitioners willing and able to deliver? A survey of attitudes to and involvement in health promotion and lifestyle counselling. Brit J General Pract 1999;49:187-90.

[10] Coleman T, Wilson A. Anti-smoking advice in general practice consultations: general practitioners' attitudes, reported practice and perceived problems. Brit J General Pract 1996;46:87-91.

[11] Rogers WA. Are guidelines ethical? Some considerations for general practice. Brit J General Pract 2002;52:663-9.

[12] Coleman T. Smoking cessation: integrating recent advances into clinical practice. Thorax 2001;56:579-82.

[13] Butler CC, Pill R, Stott NC. Qualitative study of patients' perceptions of doctors' advice to quit smoking: implications for opportunistic health promotion. $\mathrm{Br}$ Med J 1998;316:1878-81.

[14] Marinker M, Shaw J. Not to be taken as directed. Br Med J 2003;326:348-9.

[15] Grimshaw JM, Russell IT. Achieving health gain through clinical guidelines II. Ensuring guidelines change practice. Qual Health Care 1994;3:45-52.

[16] Khunti K, Lakhani MK. Barriers to the implementation of guidelines in general practice. Asthma Gen Pract 1998;6: 7-8.

[17] Kitzinger J. Qualitative research. Introducing focus groups. Br Med J 1995;311:299-302.

[18] Mays N, Pope C. Rigour and qualitative research. Br Med J 1995;311:109-12.

[19] Greenhalgh T, Taylor R. How to read a paper. Papers that go beyond numbers (qualiative research). Br Med J 1997;315:740-3.

[20] Coleman T, Murphy E, Cheater F. Factors influencing discussion of smoking between general practitioners and patients who smoke: a qualitative study. Brit J General Pract 2000;50:207-10.

[21] Coleman T, Wilson A. Anti-smoking advice from general practitioners: is a population-based approach to advice-giving feasible? Brit J General Pract 2000;50: $1001-4$.

[22] Butler CC, Rollnick S, Cohen D, Bachman M, Russell I, Stott N. Motivational consulting versus brief advice for smokers in general practice: a randomized trial. Brit J General Pract 1999;49:611-6.

[23] Young JM, Ward JE. Implementing guidelines for smoking cessation advice in Australian general practice: Opinions, current practices, readiness to change and perceived barriers. Family Practice 2001;18:14-20.

[24] Beich A, Gannik D, Malterud K. Screening and brief intervention for excessive alcohol use: qualitative interview study of the experiences of general practitioners. Br Med J) 2002;325:870.

[25] National Institute for Clinical Excellence. Guidance on the use of nicotine replacement therapy (NRT) and bupropion for smoking cessation. 2002.

[26] Lancaster T, Stead L, Silagy C, Sowden A. Effectiveness of interventions to help people stop smoking: findings from the Cochrane Library. Br Med J 2000;321:355-8.

Available online at www.sciencedirect.com science $\mathcal{Q}$ Direct. 